\title{
REVIEW
}

\section{Linear ubiquitination: A novel NF-kB regulatory mechanism for inflammatory and immune responses by the LUBAC ubiquitin ligase complex}

\author{
Fuminori Tokunaga ${ }^{1)}$ and Kazuhiro Iwai $^{2)}$ \\ ${ }^{1)}$ Laboratory of Molecular Cell Biology, Institute for Molecular and Cellular Regulation, Gunma University, Maebashi 371-8512, Japan \\ ${ }^{2)}$ Department of Molecular and Cellular Physiology, Graduate School of Medicine, Kyoto University, Kyoto 606-8501, Japan
}

\begin{abstract}
The NF- $\mathrm{kB}$ pathway is a central signaling pathway for inflammatory and immune responses, and aberrant NF$\kappa \mathrm{B}$ signaling is implicated multiple disorders, such as cancer and autoimmune, chronic inflammatory and metabolic diseases. NF- $\mathrm{\kappa B}$ is regulated by various post-translational modifications, including phosphorylation and multiple ubiquitinations. We determined that LUBAC (linear ubiquitin chain assembly complex), composed of SHARPIN, HOILIL and HOIP, generates a novel type of Met1-linked linear polyubiquitin chain and specifically regulates the canonical NF$\kappa \mathrm{B}$ pathway via the linear ubiquitination of NEMO and RIP1. In the absence of LUBAC components, NF- $\kappa \mathrm{B}$ signaling was attenuated and induced apoptosis and inflammation. Many studies on the pathophysiological functions of LUBAC, such as in B cell development, innate immune response, carcinogenesis, and osteogenesis, have been performed recently. This review summarizes these new findings on LUBAC- and linear ubiquitination-mediated NF- $\mathrm{KB}$ regulation and their implications in disorders.
\end{abstract}

Key words: Ubiquitin, NF- $\kappa \mathrm{B}$, Inflammation, Immunity

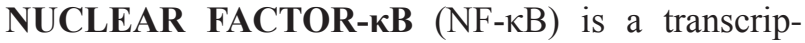
tion factor composed of homo- or heterodimers of Rel homology domain-containing proteins, including p65 (RelA), RelB, c-Rel, p105/p50 (NF-kB1) and p100/p52 (NF-kB2) $[1,2]$. The transcription factor is activated by multiple stimuli, including proinflammatory cytokines, pathogens, genotoxic agents, and oxidative stress, and the intranuclear translocation of activated NF- $\kappa B$ induces the expression of numerous genes involved in innate and adaptive immune regulation, inflammatory responses, cell adhesion, osteogenesis, and anti-apoptosis $[1,2]$. Therefore, impairments of NF- $\kappa \mathrm{B}$ regulation are closely associated with various diseases, such as cancer, rheumatoid arthritis, atherosclerosis, inflammatory bowel disease, systemic inflammatory response

Submitted Apr. 16, 2012; Accepted Apr. 19, 2012 as EJ12-0148 Released online in J-STAGE as advance publication May 19, 2012

Correspondence to: Fuminori Tokunaga, Ph.D., Laboratory of Molecular Cell Biology, Institute for Molecular and Cellular Regulation, Gunma University, Maebashi 371-8512, Japan.

E-mail: ftokunaga@gunma-u.ac.jp

Abbreviations: LUBAC, linear ubiquitin chain assembly complex; HOIL-1, heme-oxidized IRP2 ligase-1; HOIP, HOIL-1Linteracting protein syndrome, septic shock, and metabolic disease, including diabetes [1,3-5]. The intracellular NF- $\mathrm{kB}$ signaling pathway is regulated by a variety of posttranslational modifications, including phosphorylation and multiple ubiquitinations $[1,2,6]$. The present review provides a summary of a novel type of linear polyubiquitinationmediated NF- $\kappa \mathrm{B}$ regulation and its implications for immune regulation and inflammatory diseases.

\section{The Ubiquitin System}

Ubiquitin is a highly conserved, 76-amino acid (8.6 $\mathrm{kDa})$ globular protein, which is covalently conjugated to the Lys residues of targeted cellular proteins (Fig. 1). The ubiquitin system functions as a pivotal post-transcriptional modification in numerous cellular functions, including protein degradation, membrane trafficking, DNA repair, and signal transduction [7, 8]. Ubiquitination is catalyzed by three kinds of enzymes: a ubiquitin activating enzyme (E1), a ubiquitin-conjugating enzyme (E2), and a ubiquitin ligase (E3) (Fig. 2) [9]. First, the C-terminus of ubiquitin is activated by E1, using the high energy produced by ATP to AMP hydrolysis, and then is conjugated to the active site Cys resi- 
due by a reactive thioester bond. The ubiquitin is subsequently transferred to an active Cys of E2. E3 selectively recognizes both substrates and $\mathrm{E} 2$, and catalyzes ubiquitin transfer from E2 to mostly the $\varepsilon-\mathrm{NH}_{2}$ group of Lys in target proteins, via an isopeptide bond. Finally, deubiquitinases remove the ubiquitins from the target proteins, and the ubiquitins are recycled. The human
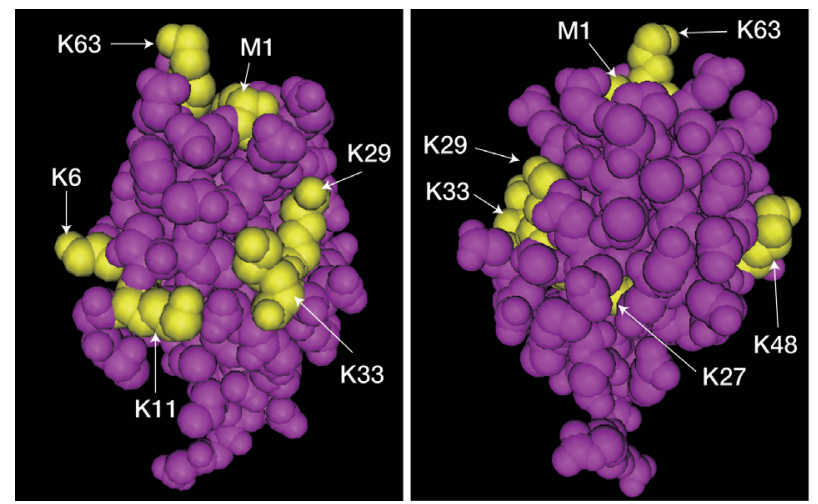

Fig. 1 Structure of ubiquitin

The seven Lys residues of ubiquitin (K6, K11, K27, K29, K33, K48, and K63) and the N-terminal Met residue (M1), which can generate polyubiquitin chains, are shown in yellow. genome encodes two E1s, approximately $30 \mathrm{E} 2 \mathrm{~s}, \sim 600$ $\mathrm{E} 3 \mathrm{~s}$, and $\sim 100$ deubiquitinases for ubiquitin, and the E3s play crucial roles in the spatiotemporal-specific recognition of the target proteins for ubiquitination.

Ubiquitination was initially characterized as a trigger for ATP-dependent intracellular proteolysis [10], however, ubiquitination is now known to function in a wide variety of cellular functions, due to its multiple linkage modes. For example, monoubiquitination and multi-monoubiquitinations of targets function in membrane trafficking and the endocytic pathway [11]. Moreover, the conjugation of polyubiquitin chains to proteins, which is possibly mediated by repeated cycles of E1, E2, and E3 activities, is important for the regulation of many proteins. Ubiquitin contains seven internal Lys residues (K6, K11, K27, K29, K33, K48 and K63) (Fig. 1), and polyubiquitination chains can be linked to any of these Lys residues [12]. Among them, the K48-linked polyubiquitin chain is known to serve as a degradation signal recognized by the $26 \mathrm{~S}$ proteasome [10], whereas the K63-linked polyubiquitin chain functions in signaling and DNA repair, without inducing degradation (Fig. 2) [13, 14]. The K11-linked

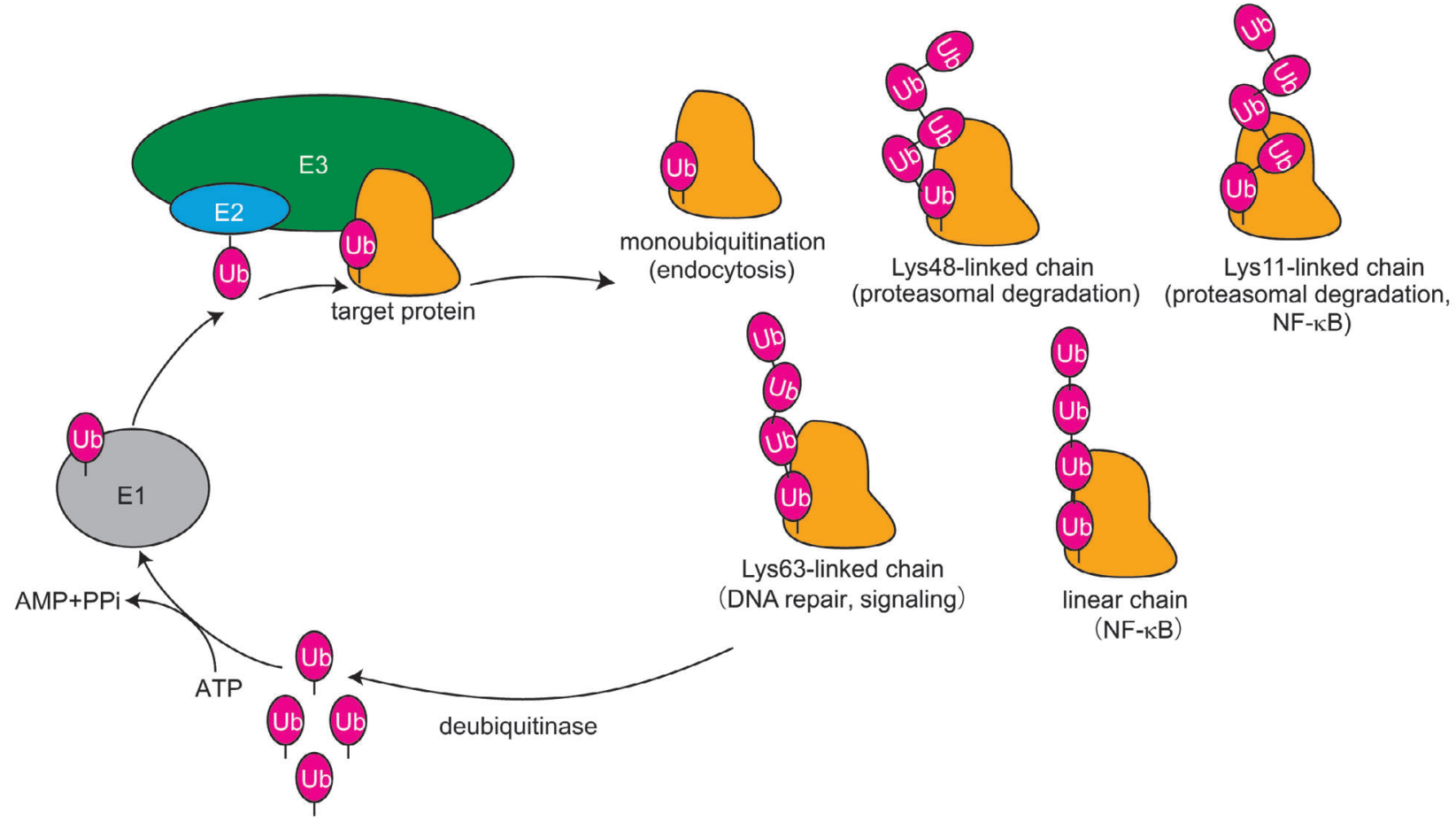

Fig. 2 The ubiquitin system and its cellular functions

Ubiquitin is conjugated to target proteins via E1 (ubiquitin activating enzyme), E2 (ubiquitin-conjugating enzyme), and E3 (ubiquitin ligase) activities. Ubiquitination of proteins regulates various cellular functions, depending on the type of linkage and the number of conjugated ubiquitins. Finally, ubiquitins are removed by deubiquitinases and recycled. 
polyubiquitin chain was initially characterized as a trigger for proteasomal degradation, but a recent report described the involvement of the chain in NF- $\mathrm{KB}$ signaling [15]. Moreover, we identified a novel type of N-terminal Met1-linked linear polyubiquitination that specifically functions in the regulation of the canonical NF-кB pathway.

\section{NF-кB Signaling Pathways}

NF- $\mathrm{kB}$ includes five Rel family transcription factors, which were discovered by David Baltimore's group in 1986. Under basal conditions, NF- $\kappa \mathrm{B}$ proteins are sequestered in the cytoplasm by inhibitory proteins (inhibitors of NF- $\kappa \mathrm{B}, \mathrm{I} \kappa \mathrm{Bs}$ ), which prevent the nuclear translocation of NF- $\mathrm{KB}$. Various stimuli induce the activation of I $\mathrm{K}$ kinase (IKK), a central kinase in the $\mathrm{NF}-\kappa \mathrm{B}$ pathway, and the phosphorylation of the I $\mathrm{kBs}$ by
IKK promotes the nuclear translocation of the transcription factor $[1,2]$. Typically, NF- $\mathrm{KB}$ activation is mediated by two pathways, the canonical and non-canonical pathways (Fig. 3). In the canonical NF- $\mathrm{kB}$ pathway, proinflammatory cytokines, such as TNF- $\alpha$ and IL-1 $\beta$, and pathogen-associated molecular patterns (PAMPs), such as bacterial lipoprotein and lipopolysaccharide (LPS), activate the canonical IKK, composed of the kinase subunits IKK $\alpha$ and IKK $\beta$ and a regulatory subunit of NEMO (NF-kB essential modulator, also known as IKK $\gamma$ ), which phosphorylates IKBs, inducing proteasomal degradation of the inhibitory proteins. The

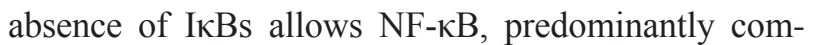
posed of $\mathrm{p} 65$ and $\mathrm{p} 50$, to enter the nucleus and activate various stimulus-specific genes (Fig. 3) [1, 2].

In contrast to the canonical pathway, the non-canonical pathway is activated relatively slowly by a subset of TNF superfamily ligands, such as lymphotoxin

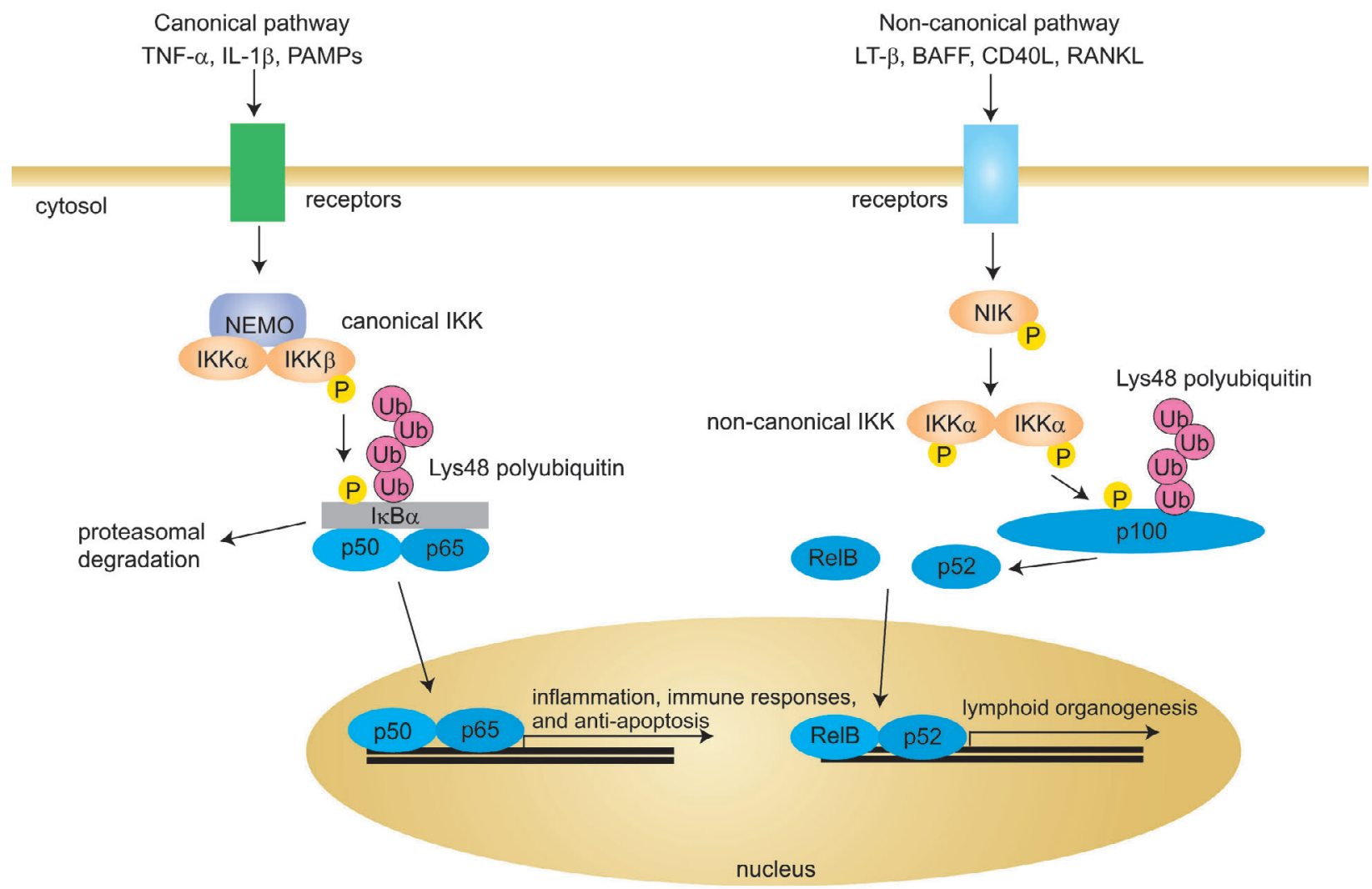

Fig. 3 The canonical and non-canonical NF- $\kappa B$ activation pathways

The canonical pathway is activated by inflammatory cytokines and PAMPs, resulting in the activation of the canonical IKK complex composed of IKK $\alpha$, IKK $\beta$ and NEMO. IKK phosphorylates IKBs, triggering their ubiquitination and proteasomal degradation, thus releasing canonical $\mathrm{NF}-\kappa \mathrm{B}$, consisting of $\mathrm{p} 50$ and $\mathrm{p} 65$, and allowing it to translocate into the nucleus and activate the expression of various genes. In contrast, the activation of the non-canonical pathway involves the activation of the IKK $\alpha$ dimer, which phosphorylates p100, promoting its ubiquitin-proteasome processing of $\mathrm{p} 52$. The activated non-canonical $\mathrm{NF}-\kappa \mathrm{B}$, which typically consists of p52 and RelB, is translocated into the nucleus. 
(LT)- $\beta$, B-cell activating factor (BAFF), and the CD40ligand. After stimulation by these ligands, the NF- $\mathrm{kB}$ inducing kinase (NIK) is stabilized, which activates the non-canonical IKK, composed of an IKK $\alpha$ dimer. The phosphorylation of p100 by the non-canonical IKK promotes the processing of $\mathrm{p} 100$ to $\mathrm{p} 52$ by the ubiquitin-proteasome system, which together with RelB regulates a subset of target genes involved in several biological phenomena, including B lymphocyte survival and lymphoid organogenesis (Fig. 3) [1, 2].

The activation of the canonical NF- $\kappa B$ pathway requires the K63-linked polyubiquitinations of NEMO and RIP1, which are mediated by Ubc13-Uev1a, an essential E2 for K63-linked ubiquitination, and the E3s of cellular inhibitor of apoptosis protein (c-IAP) and TNF receptor-associated factor (TRAF) [6]. The K63-polyubiquitinated proteins then recruit kinase-ubiquitin adaptor complexes, such as the TAK1 (TGF $\beta$-activated kinase 1)-TAB1TAB2/3 complex, to activate canonical IKK. We found that not only the K63-linked polyubiquitin chain, but the Met1-linked linear ubiquitin chain are involved in the regulation of the canonical NF- $\kappa$ B pathway.

\section{LUBAC, Composed of HOIL-1L, HOIP, and SHARPIN, Forms a Linear Polyubiquitin Chain}

We identified the LUBAC ubiquitin ligase complex, composed of HOIL-1L (heme-oxidized IRP2 ligase-1, also known as RBCK1), HOIP (HOIL-1L-interacting protein, also known as RNF31, ZIBRA, and PAUL), and SHARPIN (SHANK-associated RH domain interacting protein) (Fig. 4a). LUBAC forms a $600 \mathrm{kDa}$ physiological ternary complex, and the complex is the only E3 that assembles linear polyubiquitin chains in which the C-terminal Gly76 of one ubiquitin is linked to the $\alpha-\mathrm{NH}_{2}$ group of Met1 of another ubiquitin moiety by a peptide bond. LUBAC and its linear polyubiquitination activity are involved in NF- $\mathrm{KB}$ regulation. The characterization of each LUBAC subunit is described below.

\section{HOIL-1L}

We initially identified the $53 \mathrm{kDa}$ form of HOIL-1 as an E3 for oxidized iron regulatory protein 2 [16, 17]. Although the protein was identified as a protein kinase C-binding protein (RBCK1) [18, 19], a hepatitis $B$ virus $X$ protein $(\mathrm{HBx})$ binding protein (XAP3) [20], and an E3 for transcriptional factors [21, 22], the physiological function of the protein remained elusive. HOIL-1 contains ubiquitin-like (UBL), Npl4-type zinc finger (NZF) and RING-IBR (in-between RING)RING (RBR) domains [23]. We found that the predominant intracellular form of HOIL-1 possesses an extended $\mathrm{N}$-terminal region, due to alternative mRNA splicing, and designated the longer form $(57 \mathrm{kDa})$ of HOIL-1 as HOIL-1L. Since HOIL-1L eluted in the high molecular weight (about $600 \mathrm{kDa}$ ) fractions in the size exclusion analysis, we speculated that HOIL-1L may associate with other protein(s) to contribute to a novel function. We subsequently identified a $123 \mathrm{kDa}$ HOIL-1L-interaction protein named HOIP, by immunoprecipitation and mass spectrometry analyses [24].

\section{HOIP}

HOIP contains three zinc fingers, of which two are NZF types, as well as a ubiquitin-associated (UBA) domain and an RBR domain [24] (Fig. 4a). The UBL of HOIL-1L and the UBA of HOIP are indispensable for complex formation [24], and a unique UBL-UBA interaction mode was revealed recently [25] (Fig. 4b). The RBR domain of HOIP, but not that of HOIL-1L, is responsible for the $\mathrm{E} 3$ activity when these two proteins form a complex. The HOIL-1L-HOIP complex generates polyubiquitin chains in the presence of several E2s, including E2-25K, UbcH5s, and $\mathrm{UbcH} 7$. To identify the type of polyubiquitin linkage generated by HOIL1L-HOIP, in vitro ubiquitination assays were performed using ubiquitin mutants with Lys residues replaced by Arg. Surprisingly, polyubiquitin chain formation by HOIL-1L-HOIP was not affected by the Lys-less (K0)ubiquitin, suggesting that the ubiquitin Lys residues are dispensable for polyubiquitination by the complex. A mass spectrometric analysis indeed revealed that the C-terminal Gly76 of one ubiquitin was cross-linked to the $\alpha-\mathrm{NH}_{2}$ group of Met1 of another ubiquitin moiety to form a novel type of polyubiquitin chain, linear polyubiquitin. In the tertiary structure of ubiquitin, Met1 and Lys63 are located close to each other (Fig. 1), and the linear and K63-linked ubiquitin chains adopt similar conformations, although they have distinct physiological roles (Fig. 4c). The complex was therefore designated as LUBAC (linear ubiquitin chain assembly complex) [24]. At present, LUBAC is the only E3 that generates a linear polyubiquitin chain, and the linkage specificity is defined by LUBAC, and not by the E2s. Recently, some of the RBR family E3s, such as parkin and ariadne, were reported to function in a RING/ 
HECT hybrid reaction [26]; i.e., they bind E2s via the RING1 domain and transfer them to a conserved Cys residue in the RING2 domain by a thioester-linkage, similar to the HECT-type E3s. Although LUBAC may utilize a similar reaction mode, the precise molecular mechanism by which it forms linear-specific polyubiquitin chains is still unknown.

\section{SHARPIN}

SHARPIN was first identified as a protein that bound Shank1, a postsynaptic density-enriched protein [27]. Although SHARPIN is expressed ubiquitously, its physiological function remains unknown. Interestingly, the C-terminal portion of SHARPIN shares significant sequence similarity with the $\mathrm{N}$-terminal region of HOIL-1L (Fig. 4a). A hint as to the possible function of SHARPIN was provided by the identification of a mouse Sharpin gene mutation responsible for the molecular pathogenesis of spontaneous chronic proliferative dermatitis in mice (cpdm) (Fig. 4d) [28].

$C p d m$ mice were initially characterized as autosomal recessive mutants with severe chronic inflammatory skin lesions at 3 to 5 weeks [29-33]. The mice develop psoriasis-like proliferative skin lesions, such as erythema, hair loss, epidermal hyperplasia, multifocal parakeratosis and hyperkeratosis, with the infiltration of granulocytes, macrophages, and eosinophils within the dermis and epidermis. In addition to dermatitis, the mice exhibit splenomegaly, defects in secondary lymphoid organs, such as the absence of Peyer's patches and the marginal zone in the spleen, elevated mast cell-bound IgE but significantly decreased serum levels of $\operatorname{IgG}, \operatorname{IgA}$, and $\operatorname{IgE}$, defective TH1 cytokine production, and decreased bone mineral content and a

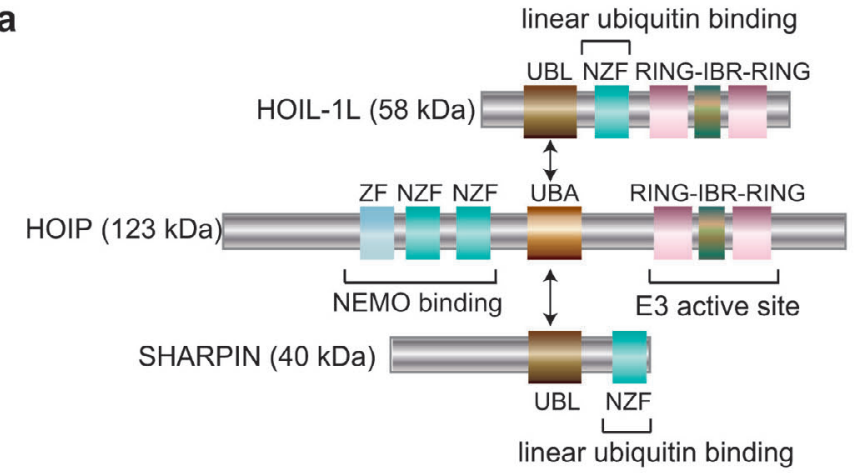

C

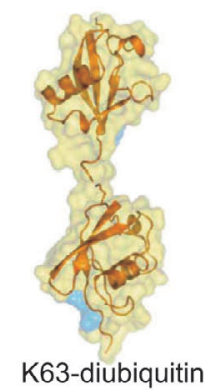

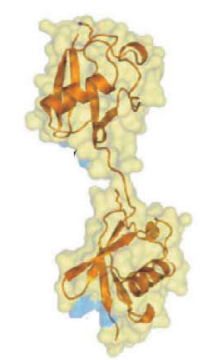

linear diubiquitin d

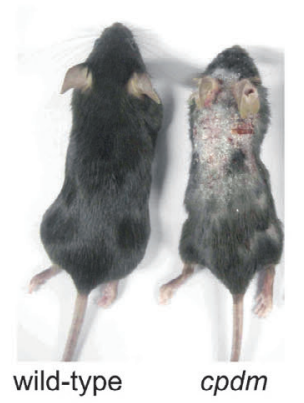

b

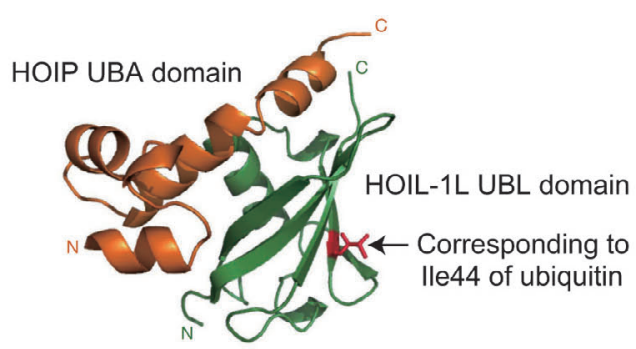

e

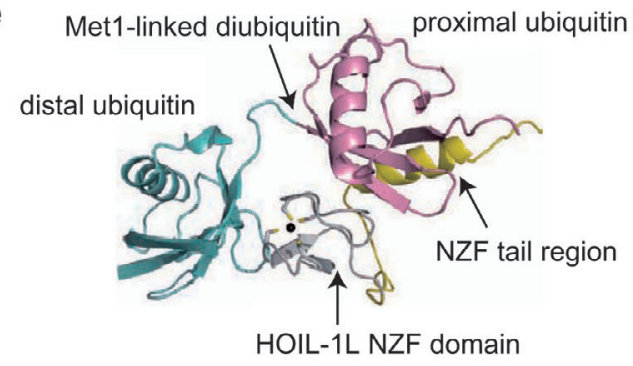

Figs. 4b, c, and e are reproduced with permissions from Refs. [25], [43], and [39], respectively.

Fig. 4 Structure and function of LUBAC

(a), Domain structures of HOIL-1L, HOIP, and SHARPIN. UBL, ubiquitin-like; NZF, Npl4-type zinc finger; RING, really interesting new gene; IBR, in-between RING; ZF, zinc finger; UBA, ubiquitin-associated domains. The zinc fingers and the RING domains of HOIP are the substrate-binding site and the E3 active site, respectively. The NZF domains in HOIL-1L and SHARPIN specifically bind linear ubiquitin. (b), The non-canonical UBA-UBL interaction between HOIP and HOIL-1L. The crystal structure of the HOP UBA-HOIL-1L UBL complex, reported by Kato and co-workers [25], is shown. The Ile44 hydrophobic patch in UBL, which is the canonical interaction site for UBA, is not involved in the interaction. (c), K63-linked diubiquitin and linear diubiquitin adopt similar conformations [43]. (d), Phenotypes of wild-type and cpdm mice. Sharpinablated $c p d m$ mice show severe skin lesions, eosinophil infiltration, and defects in secondary lymphoid organs. (e), Structure of the linear-diubiquitin-HOIL-1L NZF complex (modified from [39]). HOIL-1L NZF specifically recognizes distal and proximal linear ubiquitins, but not other linkages. 
density [34]. Positional cloning studies from two independent $c p d m$ mouse strains, C57BL/KaLawRij-cpdm/ $c p d m$ and CBy.OcB3-cpdm ${ }^{D e m} / c p d m^{D e m}$, revealed mutations in exon 1 of the Sharpin gene, resulting in a frameshift and the premature termination of the protein [28]. Thus, the absence of SHARPIN is responsible for the various $c p d m$ phenotypes described above. However, the mechanism by which the lack of SHARPIN results in these phenotypes remained unclear, until recently. We and two other groups discovered that SHARPIN is involved as a physiological component of LUBAC [35-37], although the detailed stoichiometry of SHARPIN, HOIL-1L, and HOIP in LUBAC has not been determined yet.

\section{LUBAC Regulates the Canonical NF-кB Pathway by Linear Ubiquitination}

\section{The involvement of linear ubiquitination in $\mathrm{NF}-\boldsymbol{\kappa} B$ regulation}

We first determined that the overexpression of LUBAC specifically induced NF- $\mathrm{KB}$ activity, by a luciferase reporter assay [38]. Conversely, the knockdown of LUBAC components resulted in reduced basal and TNF- $\alpha$-stimulated NF- $\kappa B$ activities. Moreover, an overexpression study revealed that the NF- $\kappa \mathrm{B}$ complex activated by LUBAC consists of $\mathrm{p} 50$ and $\mathrm{p} 65$, suggesting that LUBAC is involved in the activation of the canonical NF-KB pathway [38]. The E3 activity of HOIP to generate linear polyubiquitin is required for the efficient activation of canonical IKK, and the subsequent phosphorylation of I $\mathrm{I} B \alpha$ suggested that LUBAC targets components of the canonical IKK pathway or molecules functioning upstream from IKK in the $\mathrm{NF}-\mathrm{\kappa B}$ activation pathway. Further analyses revealed that the association between the coiled-coil2 leucine zipper (CC2-LZ) domains of NEMO and the zinc finger domains of HOIP leads to the LUBAC-mediated linear ubiquitination of NEMO at Lys285 and Lys309. The replacement of these Lys residues with Arg suppressed the linear polyubiquitination of NEMO and the LUBAC- and inflammatory cytokine-induced NF- $\mathrm{kB}$ activation [38]. The deletion of the NZF domains of HOIL-1L and SHARPIN caused a significant reduction of the NF- $\kappa$ B activity. Recently, the NZF domain of HOIL-1L was shown to bind linear ubiquitin specifically (Fig. 4e) [39], suggesting that LUBAC-mediated production and association with linear ubiquitin are necessary for sufficient NF- $\kappa \mathrm{B}$ activation.
Interestingly, NEMO and ABINs (A20 binding and inhibitor of NF- $\kappa B$ ) contain a ubiquitin binding site named UBAN (also known as NOA or NUB) within the CC2-LZ domain, and the UBAN domain of NEMO exhibits 100-fold higher affinity for linear di-ubiquitin than for K63- and K48-linked di-ubiquitin [40, 41]. The crystal structure of NEMO UBAN revealed an $\alpha$-helical structure and a dimer that specifically recognizes linear di-ubiquitin [40, 41]. Importantly, mutations within the linear ubiquitin binding site of NEMO (D311N and D311G) reportedly induced X-linked anhidrotic ectodermal dysplasia with immunodeficiency (EDA-ID) [42], indicating that the UBAN domain is critical for NEMO function, possibly because it recognizes linear polyubiquitin chains. Thus, although linear and K63linked ubiquitin adopt similar conformations [43], these ubiquitin chains are recognized by distinct components of the NF- $\kappa \mathrm{B}$ activation pathway, and they both play central roles in NF- $\mathrm{\kappa B}$ activation.

\section{LUBAC is a c-IAPs-dependent regulator in the TNF receptor superfamily}

TNF- $\alpha$ is a critical inflammatory cytokine in the induction of canonical NF- $\mathrm{KB}$ activation for cell survival. TNF- $\alpha$ binds to TNF receptor 1 and activates a signaling complex (complex I), composed of receptor interacting kinase 1 (RIP1), TNFR1-associated death domain (TRADD), TRAF2, and c-IAP1/2 [44]. Subsequently, complex I dissociates from the receptor, and FADD and caspase 8 are recruited to form complex II for apoptosis induction. LUBAC becomes a component of the TNF receptor 1 signaling complex upon stimulation with TNF- $\alpha$, and the recruitment of LUBAC to TNF receptor 1 depends on TRADD, TRAF2, and c-IAP-1/2, but does not require either RIP1 or NEMO (Fig. 5a) $[45,46]$. Smac mimetics, which bind c-IAPs and induce auto-ubiquitination and degradation of the ubiquitin ligases, affect the recruitment of LUBAC to various TNF receptor superfamily proteins [46]. Interestingly, TRAF-mediated K63-linked ubiquitination of RIP1 is followed by proteasomal degradation via the addition of K48-linked ubiquitin chains by A20 [47]. In addition to these polyubiquitination functions, c-IAPs and LUBAC promote the K11-linked and linear ubiquitinations of RIP1 [15], respectively. Thus, various types of ubiquitin chain modifications seem to be required at the initial step of NF- $\mathrm{KB}$ activation (Fig. 5a). Further analyses will clarify the distinct roles of these polyubiquitin chains. 
a

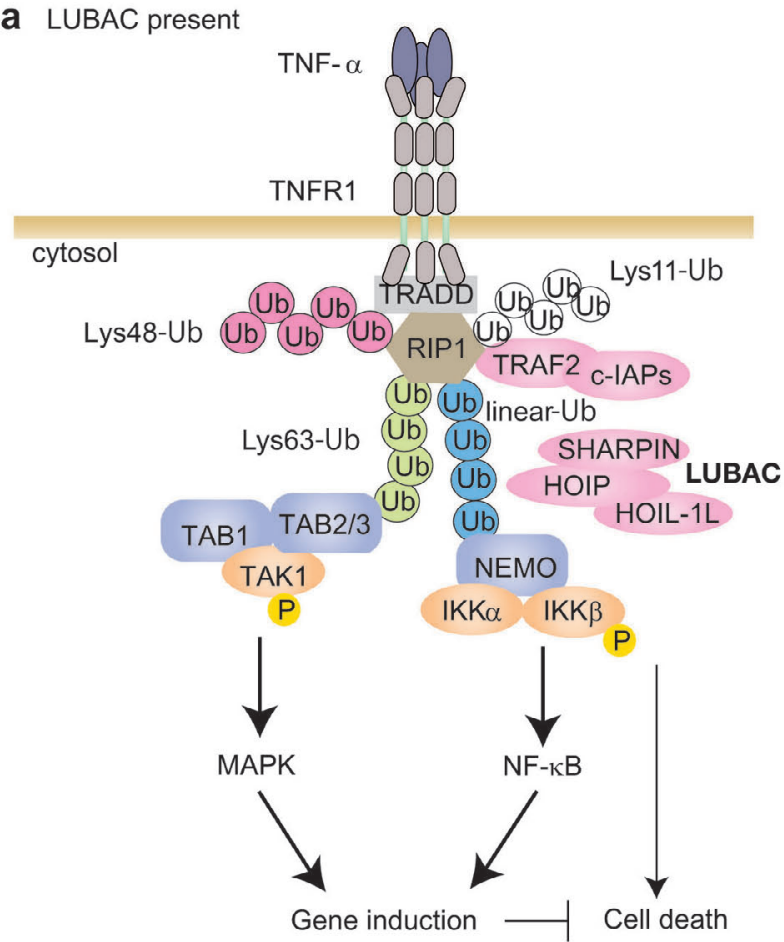

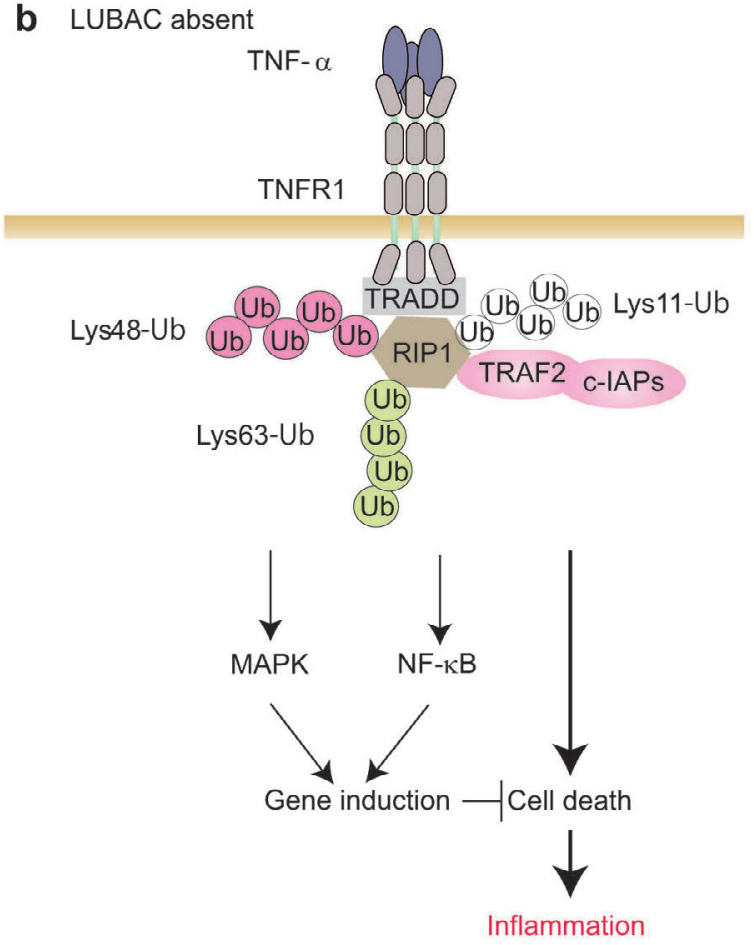

Fig. 5 Schematic diagram showing LUBAC-mediated canonical NF- $\kappa$ B regulation In the presence of LUBAC (left panel), proinflammatory cytokines, such as TNF- $\alpha$, trigger the recruitment of TRAF2 and c-IAPs to the cytosolic portion of TNF receptor 1, associated with TRADD and RIP1, to form complex I. RIP1 accepts multiple types of ubiquitination and induces LUBAC recruitment. Linear ubiquitination of NEMO and RIP1 by LUBAC activates the canonical IKK for NF- $\mathrm{B}$ activation. In addition to the activation of MAP kinases initiated by TAK1-TAB1-TAB2/3, gene induction prevents cell death. In contrast, in the absence of LUBAC components (right panel), TNF- $\alpha$ induced gene expression is attenuated, and complex I is destabilized to form complex II, which induces cell death by apoptosis and necroptosis.

\section{Genetic ablation of LUBAC components induces apoptosis and inflammation}

HOIP includes an active E3 site, and both HOIL-1L and SHARPIN are accessory proteins with similar domain organizations (Fig. 4a). HOIL- $1 L^{-/-}$and Sharpin-deficient $c p d m$ mice are defective in NF- $\mathrm{BB}$ activation, due to the decreased amount of the 600 $\mathrm{kDa}$ ternary LUBAC complex in cells [35-38]. Cells derived from both $c p d m$ mice and $H O I L-1 L^{-/-}$mice show decreased activation of the canonical NF- $\mathrm{kB}$ pathway in response to TNF- $\alpha$, IL-1 $\beta$, CD40-ligand, lipopolysaccharide (LPS), and LT- $\beta$ stimulation, which did not significantly affect the non-canonical NF- $\kappa B$ pathway [35-38]. TNF- $\alpha$ stimulation induced rapid apoptosis of $H O I L-1 L^{-/-}$and $c p d m$ cells via FADD- and caspase 8-dependent pathways, resulting in the activation of caspase 3 (Fig. 5b). These results strongly indicated that LUBAC plays crucial roles in the activation of the canonical NF- $\kappa$ B pathway.

However, unlike $c p d m$ mice, HOIL-1L $L^{-/-}$mice do not exhibit overt phenotypes. The TNF- $\alpha$-induced apoptosis in cpdm cells is more severe than that in $H O I L-1 L^{-/-}$cells [35]. Interestingly, the ablation of at least one TNF- $\alpha$ allele prevented skin lesion formation in cpdm mice, although the depletion of TNF- $\alpha$ had no effect on the immunological phenotype [36]. These results suggested that the impairment of signal-induced NF- $\kappa \mathrm{B}$ activation per se does not provoke the severe phenotypes found in $c p d m$ mice. A recent study showed that SHARPIN inhibits $\beta 1$-integrin function through direct binding to the cytoplasmic region of integrin $\alpha$-subunits, thus blocking the recruitment of talin and kindlin to integrin [48]. In cpdm mice, the $\beta 1$-integrin activity was significantly increased in keratin 14-positive keratinocytes, splenocytes, and bonemarrow leukocytes. The ubiquitin-independent binding of SHARPIN to integrin in the absence of HOIP and HOIL-1L, along with the fact that the silencing of HOIP did not induce $\beta 1$-integrin activity in cells, implies that the free form of SHARPIN is fully func- 
tional in integrin regulation. Therefore, in addition to HOIL-1L, SHARPIN may also play an important role in $\beta 1$-integrin inhibition, cell adhesion and migration, and its loss may be one of the underlying causes of the dermatitis in Sharpin-deficient cpdm mice.

\section{Pathophysiological Functions of LUBAC}

The linear polyubiquitination activity of LUBAC is a critical regulator of the canonical NF- $\mathrm{KB}$ pathway. Currently, the number of pathophysiological phenomena in which LUBAC plays a role is rapidly expanding.

\section{$L U B A C$ regulates $B$ cell function}

The LUBAC components of SHARPIN, HOIL-1L, and HOIP are expressed ubiquitously, but they are abundantly expressed in thymus and spleen, suggesting that LUBAC functions in lymphocytes [35]. CD40, a member of the TNF receptor superfamily of $B$ cells, binds the CD40-ligand (CD154), which is transiently expressed by $\mathrm{T}$ cells and other cells under inflammatory conditions [49]. CD40 stimulation activates both the canonical and non-canonical NF- $\mathrm{kB}$ pathways, and the signaling is necessary for isotype switching, high affinity antibody production, and the development of humoral immunological memory. LUBAC is recruited to the $\mathrm{CD} 40$ receptor-signaling complex in response to stimulation, and the CD40-induced canonical NF-kB activation, but not the non-canonical pathway, is attenuated in B cells derived from $c p d m$ and $H O I L-1 L^{-/-}$ mice $[35-38,50]$. Furthermore, the ablation of HOIP in a B cell line impaired CD40 signaling and abolished the recruitment of the IKK complex to CD40 [51]. Collectively, these results indicated that LUBAC and its linear ubiquitination activity are indispensable for CD40 signaling and B cell functions.

\section{LUBAC is involved in the innate immune response}

The innate immune system protects humans from microbial infection by recognizing microbial specific molecular patterns (PAMPs), which are sensed by various pattern recognition receptors, such as Tolllike receptors (TLR) and retinoic acid-inducible gene (RIG)-I-like receptors [52]. Stimulation of these signaling receptors induces NF- $\mathrm{KB}$ activation and type I interferon production. LPS in Gram-negative bacteria, which causes septic shock, is recognized by TLR4 with myeloid differentiation factor 2 (MD2). In Sharpinablated cpdm macrophages, LPS-induced NF- $\kappa \mathrm{B}$ acti- vation is attenuated [37], suggesting that LUBAC is involved in the activation of the innate immune system. Moreover, the depletion of SHARPIN in cpdm macrophages strongly attenuated the production of IL-12p40 in response to various TLR ligands, including $\mathrm{Pam}_{3} \mathrm{CSK}_{4}$ for TLR2 and infection with Listeria monocytogene [53]. Therefore, LUBAC seems to play a role in the TLR signaling pathway.

Furthermore, LUBAC negatively regulates RIG-Iand TRIM25-mediated type I interferon induction $[54,55]$. RIG-I is localized in the cytoplasm, where it recognizes dsRNA from viruses [56]. The generation of K63-linked ubiquitin by the E3 ubiquitin ligase TRIM25 and the subsequent association with the mitochondrial adaptor protein MAVS (also known as IPS-1, VISA, or Cardif) trigger RIG-I activation $[57,58]$. The NZF domain of HOIL-1L competes with TRIM25 for RIG-I binding, and LUBAC facilitates the proteasomal degradation of TRIM25, suggesting that LUBAC may suppress interferon-mediated antiviral signaling [55]. Moreover, the overexpression of HOIL-1L reportedly induced the proteasomal degradation of interferon regulatory factor (IRF) 3 [54], although IRF3 may not be a direct substrate of LUBAC [55]. Thus, LUBAC seems to be a crucial regulator of the innate immunity response, by modulating the $\mathrm{NF}-\mathrm{\kappa B}$ and interferon production pathways.

\section{LUBAC functions in carcinogenesis, and is activated by anti-cancer agents}

Constitutive activation of NF- $\mathrm{\kappa B}$ has been demonstrated in several types of cancers, although the precise mechanism underlying the NF- $\mathrm{KB}$ activation remains elusive. Interestingly, SHARPIN expression is enhanced in various tumor tissues [59], and moreover, LUBAC is involved in the metastasis of osteosarcoma cells [60]. Thus, LUBAC-induced NF- $\kappa B$ activation, resulting in the expression of ICAM-1 and other factors, may be a key regulator for carcinogenesis and metastasis.

DNA damaging genotoxic agents, including anticancer agents such as etoposide, as well as reactive oxygen species, chemicals, and radiation, induce nuclear-initiated NF- $\kappa B$ activation [61]. First, the DNA damage response activates ataxia telangiectasia mutated (ATM) kinase, which belongs to the phosphoinositide-3-kinase-related kinase family, in the nucleus. Interestingly, free NEMO, which is not bound to either IKK $\alpha$ or IKK $\beta$ in the nucleus, accepts various modifi- 
cations in this step. ATM activation induces the phosphorylation and subsequent SUMOylation of NEMO at Lys277 and Lys309 [62]. These SUMOylation sites of NEMO, together with Lys285, are mono-ubiquitinated by c-IAP, triggering the formation of a complex with IKK $\beta$ in the cytoplasm to activate NF- $\mathrm{KB}$ $[63,64]$. Importantly, these crucial Lys residues are identical to those targeted for linear ubiquitination by LUBAC, and indeed, LUBAC is required for genotoxic NF- $\kappa B$ activation [65]. LUBAC linearly ubiquitinates NEMO in the cytoplasm upon genotoxic stress, with the subsequent activation of TAK1 and IKK. Thus, LUBAC-mediated linear ubiquitination of NEMO may be involved in genotoxic NF- $\mathrm{\kappa B}$ activation, to protect cells from DNA damage-induced apoptosis.

\section{LUBAC contributes to osteogenesis}

NF- $\kappa B$ plays a pivotal role in skeletal development, by affecting osteoclasts, osteoblasts, and endochondral ossification [66]. Therefore, impaired NF- $\mathrm{kB}$ signaling is involved in a number of pathologic skeletal conditions, including osteoarthritis, rheumatoid arthritis, postmenopausal osteoporosis, Paget's disease, and metastatic bone disease. Sharpin-deficient cpdm mice show lower total and cortical bone mineral content and bone mineral density, trabecular and cortical bone volume, and trabecular number, indicating that both the osteoclasts and osteoblasts in cpdm mice are defective [67]. Furthermore, Landgraf et al. identified SHARPIN (Sipl1) and HOIL-1L (Rbck1) as the novel interaction partners of Eyal (eyes absent 1 protein). In humans, the mutation of Eya1 induces BOR (branchio-oto-renal) syndrome, characterized by kidney defects, hearing loss, and branchial arch anomalies [68]. SHARPIN and HOIL-1L enhance the transcriptional activity of the Six proteins, as co-activators with Eya. The knockdowns of SHARPIN and HOIL-1L in zebrafish caused a BOR syndrome-like phenotype, indicating that SHARPIN and HOIL-1L function in craniofacial development, whereas the contribution of HOIP is unknown. In addition to TNF- $\alpha$, RANKL (receptor activator of NF- $\mathrm{kB}$ ligand) and its receptor RANK play important roles in osteoclasts and osteoblasts [69]. Further studies are necessary to clarify the significance of LUBAC in RANK-RANKL signaling.

\section{Perspectives}

LUBAC, composed of SHARPIN, HOIL-1L, and HOIP, generates a novel type of linear polyubiquitin chain that principally regulates canonical NF- $\mathrm{KB}$ activation. Recent studies on LUBAC have revealed that the ligase plays important roles in inflammatory responses, acquired and innate immunities, lymphocyte development, interferon production, genotoxic stress response, and skeletal conditions. Therefore, LUBAC seems to be correlated with various inflammatory, infectious and autoimmune diseases, such as psoriasis-like dermatitis, rheumatoid arthritis, sepsis, and systemic lupus erythematosus. Metabolic diseases involving obesity, type 2 diabetes, and atherosclerosis are induced by chronic low-level inflammation, and NF- $\mathrm{KB}$ is a key regulator for the disorders [70]. It is quite possible that LUBAC is involved in the pathogenesis of metabolic diseases. Further studies are necessary to clarify the biological roles and pathological significance of LUBAC.

\section{Acknowledgements}

We would like to thank all of our collaborators. This work was supported by a Grant-in-Aid for Scientific Research (KAKENHI) from Ministry of Education, Culture, Sports, Science and Technology (MEXT) and Japan Society for the Promotion of Science (JSPS), Japan.

\section{Disclosure Statement}

Authors have nothing to declare.

\section{References}

1. Hayden MS, Ghosh S (2012) NF- $\kappa B$, the first quartercentury: remarkable progress and outstanding questions. Genes Dev 26: 203-234.

2. Vallabhapurapu S, Karin M (2009) Regulation and function of NF- $\mathrm{B}$ transcription factors in the immune system. Annu Rev Immunol 27: 693-733.
3. Perkins ND (2012) The diverse and complex roles of NF- $\mathrm{B}$ subunits in cancer. Nat Rev Cancer 12: 121132.

4. Ben-Neriah Y, Karin M (2011) Inflammation meets cancer, with NF- $\mathrm{KB}$ as the matchmaker. Nat Immunol 12: 715-723. 
5. Pasparakis M (2009) Regulation of tissue homeostasis by NF- $\kappa$ B signalling: implications for inflammatory diseases. Nat Rev Immunol 9: 778-788.

6. Chen ZJ (2012) Ubiquitination in signaling to and activation of IKK. Immunol Rev 246: 95-106.

7. Hershko A, Ciechanover A (1998) The ubiquitin system. Annu Rev Biochem 67: 425-479.

8. Kornitzer D, Ciechanover A (2000) Modes of regulation of ubiquitin-mediated protein degradation. J Cell Physiol 182: 1-11.

9. Pickart CM (2001) Mechanisms underlying ubiquitination. Annu Rev Biochem 70: 503-533.

10. Hershko A, Ciechanover A (1992) The ubiquitin system for protein degradation. Annu Rev Biochem 61: 761807.

11. Hicke L, Dunn R (2003) Regulation of membrane protein transport by ubiquitin and ubiquitin-binding proteins. Annu Rev Cell Dev Biol 19: 141-172.

12. Xu P, Duong DM, Seyfried NT, Cheng D, Xie Y, Robert J, Rush J, Hochstrasser M, Finley D, Peng J (2009) Quantitative proteomics reveals the function of unconventional ubiquitin chains in proteasomal degradation. Cell 137: 133-145.

13. Ashley C, Pastushok L, McKenna S, Ellison MJ, Xiao W (2002) Roles of mouse UBC13 in DNA postreplication repair and Lys63-linked ubiquitination. Gene 285: 183-191.

14. Kawadler H, Yang X (2006) Lys63-linked polyubiquitin chains: linking more than just ubiquitin. Cancer Biol Ther 5: 1273-1274.

15. Dynek JN, Goncharov T, Dueber EC, Fedorova AV, Izrael-Tomasevic A, Phu L, Helgason E, Fairbrother WJ, Deshayes K, Kirkpatrick DS, Vucic D (2010) c-IAP1 and $\mathrm{UbcH} 5$ promote K11-linked polyubiquitination of RIP1 in TNF signalling. EMBO J 29: 4198-4209.

16. Yamanaka $\mathrm{K}$, Ishikawa $\mathrm{H}$, Megumi $\mathrm{Y}$, Tokunaga $\mathrm{F}$, Kanie M, Rouault TA, Morishima I, Minato N, Ishimori K, Iwai K (2003) Identification of the ubiquitin-protein ligase that recognizes oxidized IRP2. Nat Cell Biol 5: 336-340.

17. Ishikawa $\mathrm{H}$, Kato $\mathrm{M}$, Hori $\mathrm{H}$, Ishimori $\mathrm{K}$, Kirisako $\mathrm{T}$, Tokunaga F, Iwai K (2005) Involvement of heme regulatory motif in heme-mediated ubiquitination and degradation of IRP2. Mol Cell 19: 171-181.

18. Tokunaga C, Kuroda S, Tatematsu K, Nakagawa N, Ono Y, Kikkawa U (1998) Molecular cloning and characterization of a novel protein kinase $\mathrm{C}$-interacting protein with structural motifs related to RBCC family proteins. Biochem Biophys Res Commun 244: 353-359.

19. Nakamura M, Tokunaga F, Sakata S, Iwai K (2006) Mutual regulation of conventional protein kinase $\mathrm{C}$ and a ubiquitin ligase complex. Biochem Biophys Res Commun 351: 340-347.

20. Cong YS, Yao YL, Yang WM, Kuzhandaivelu N, Seto E (1997) The hepatitis B virus X-associated protein,
$\mathrm{XAP} 3$, is a protein kinase C-binding protein. $\mathrm{J}$ Biol Chem 272: 16482-16489.

21. Bayle J, Lopez S, Iwai K, Dubreuil P, De Sepulveda P (2006) The E3 ubiquitin ligase HOIL-1 induces the polyubiquitination and degradation of SOCS6 associated proteins. FEBS Lett 580: 2609-2614.

22. Zenke-Kawasaki Y, Dohi Y, Katoh Y, Ikura T, Ikura M, Asahara T, Tokunaga F, Iwai K, Igarashi K (2007) Heme induces ubiquitination and degradation of the transcription factor Bach1. Mol Cell Biol 27: 6962-6971.

23. Eisenhaber B, Chumak N, Eisenhaber F, Hauser MT (2007) The ring between ring fingers (RBR) protein family. Genome Biol 8: 209.

24. Kirisako T, Kamei K, Murata S, Kato M, Fukumoto H, Kanie M, Sano S, Tokunaga F, Tanaka K, Iwai K (2006) A ubiquitin ligase complex assembles linear polyubiquitin chains. EMBO J 25: 4877-4887.

25. Yagi H, Ishimoto K, Hiromoto T, Fujita H, Mizushima T, Uekusa Y, Yagi-Utsumi M, Kurimoto E, Noda M, Uchiyama S, Tokunaga F, Iwai K, Kato K (2012) A non-canonical UBA-UBL interaction forms the linearubiquitin-chain assembly complex. EMBO Rep 13: 462468.

26. Wenzel DM, Lissounov A, Brzovic PS, Klevit RE (2011) $\mathrm{UBCH} 7$ reactivity profile reveals parkin and HHARI to be RING/HECT hybrids. Nature 474: 105-108.

27. Lim S, Sala C, Yoon J, Park S, Kuroda S, Sheng M, Kim E (2001) Sharpin, a novel postsynaptic density protein that directly interacts with the shank family of proteins. Mol Cell Neurosci 17: 385-397.

28. Seymour RE, Hasham MG, Cox GA, Shultz LD, Hogenesch H, Roopenian DC, Sundberg JP (2007) Spontaneous mutations in the mouse Sharpin gene result in multiorgan inflammation, immune system dysregulation and dermatitis. Genes Immun 8: 416-421.

29. HogenEsch H, Gijbels MJ, Offerman E, van Hooft J, van Bekkum DW, Zurcher C (1993) A spontaneous mutation characterized by chronic proliferative dermatitis in C57BL mice. Am J Pathol 143: 972-982.

30. Gallardo Torres HI, Gijbels MJ, HegnEsch H, Kraal G (1995) Chronic proliferative dermatitis in mice: neutrophil-endothelium interactions and the role of adhesion molecules. Pathobiology 63: 341-347.

31. Gijbels MJ, HogenEsch H, Blauw B, Roholl P, Zurcher C (1995) Ultrastructure of epidermis of mice with chronic proliferative dermatitis. Ultrastruct Pathol 19: 107-111.

32. Gijbels MJ, HogenEsch H, Bruijnzeel PL, Elliott GR, Zurcher C (1995) Maintenance of donor phenotype after full-thickness skin transplantation from mice with chronic proliferative dermatitis ( $c p d m / c p d m)$ to C57BL/ $\mathrm{Ka}$ and nude mice and vice versa. $J$ Invest Dermatol 105: 769-773.

33. Gijbels MJ, Zurcher C, Kraal G, Elliott GR, HogenEsch H, Schijff G, Savelkoul HF, Bruijnzeel PL (1996) 
Pathogenesis of skin lesions in mice with chronic proliferative dermatitis (cpdm/cpdm). Am J Pathol 148: 941950.

34. HogenEsch H, Janke S, Boggess D, Sundberg JP (1999) Absence of Peyer's patches and abnormal lymphoid architecture in chronic proliferative dermatitis ( $\mathrm{cpdm} /$ cpdm) mice. J Immunol 162: 3890-3896.

35. Tokunaga F, Nakagawa T, Nakahara M, Saeki Y, Taniguchi M, Sakata S, Tanaka K, Nakano H, Iwai $\mathrm{K}$ (2011) SHARPIN is a component of the NF- $\kappa B-$ activating linear ubiquitin chain assembly complex. Nature 471: 633-636.

36. Gerlach B, Cordier SM, Schmukle AC, Emmerich CH, Rieser E, Haas TL, Webb AI, Rickard JA, Anderton H, Wong WW, Nachbur U, Gangoda L, Warnken U, Purcell AW, Silke J, Walczak H (2011) Linear ubiquitination prevents inflammation and regulates immune signalling. Nature 471: 591-596.

37. Ikeda F, Deribe YL, Skanland SS, Stieglitz B, Grabbe C, Franz-Wachtel M, van Wijk SJ, Goswami P, Nagy V, Terzic J, Tokunaga F, Androulidaki A, Nakagawa T, Pasparakis M, Iwai K, Sundberg JP, Schaefer L, Rittinger K, Macek B, Dikic I (2011) SHARPIN forms a linear ubiquitin ligase complex regulating $\mathrm{NF}-\kappa \mathrm{B}$ activity and apoptosis. Nature 471: 637-641.

38. Tokunaga F, Sakata S, Saeki Y, Satomi Y, Kirisako T, Kamei K, Nakagawa T, Kato M, Murata S, Yamaoka S, Yamamoto M, Akira S, Takao T, Tanaka K, Iwai K (2009) Involvement of linear polyubiquitylation of NEMO in NF- $\kappa \mathrm{B}$ activation. Nat Cell Biol 11: 123132.

39. Sato Y, Fujita H, Yoshikawa A, Yamashita M, Yamagata A, Kaiser SE, Iwai K, Fukai S (2011) Specific recognition of linear ubiquitin chains by the Npl4 zinc finger (NZF) domain of the HOIL-1L subunit of the linear ubiquitin chain assembly complex. Proc Natl Acad Sci U S A 108: 20520-20525.

40. Rahighi S, Ikeda F, Kawasaki M, Akutsu M, Suzuki N, Kato R, Kensche T, Uejima T, Bloor S, Komander D, Randow F, Wakatsuki S, Dikic I (2009) Specific recognition of linear ubiquitin chains by NEMO is important for NF- $\kappa \mathrm{B}$ activation. Cell 136: 1098-1109.

41. Lo YC, Lin SC, Rospigliosi CC, Conze DB, Wu CJ, Ashwell JD, Eliezer D, Wu H (2009) Structural basis for recognition of diubiquitins by NEMO. Mol Cell 33: 602-615.

42. Hubeau M, Ngadjeua F, Puel A, Israel L, Feinberg J, Chrabieh M, Belani K, Bodemer C, Fabre I, Plebani A, Boisson-Dupuis S, Picard C, Fischer A, Israel A, Abel L, Veron M, Casanova JL, Agou F, Bustamante J (2011) New mechanism of X-linked anhidrotic ectodermal dysplasia with immunodeficiency: impairment of ubiquitin binding despite normal folding of NEMO protein. Blood 118: 926-935.

43. Komander D, Reyes-Turcu F, Licchesi JD, Odenwaelder
P, Wilkinson KD, Barford D (2009) Molecular discrimination of structurally equivalent Lys 63-linked and linear polyubiquitin chains. EMBO Rep 10: 466-473.

44. Vucic D, Dixit VM, Wertz IE (2011) Ubiquitylation in apoptosis: a post-translational modification at the edge of life and death. Nat Rev Mol Cell Biol 12: 439-452.

45. Haas TL, Emmerich CH, Gerlach B, Schmukle AC, Cordier SM, Rieser E, Feltham R, Vince J, Warnken U, Wenger T, Koschny R, Komander D, Silke J, Walczak H (2009) Recruitment of the linear ubiquitin chain assembly complex stabilizes the TNF-R1 signaling complex and is required for TNF-mediated gene induction. Mol Cell 36: 831-844.

46. Varfolomeev E, Goncharov T, Maecker H, Zobel K, Komuves LG, Deshayes K, Vucic D (2012) Cellular Inhibitors of Apoptosis Are Global Regulators of NF- $\kappa \mathrm{B}$ and MAPK Activation by Members of the TNF Family of Receptors. Sci Signal 5: ra22.

47. Wertz IE, O’Rourke KM, Zhou H, Eby M, Aravind L, Seshagiri S, Wu P, Wiesmann C, Baker R, Boone DL, Ma A, Koonin EV, Dixit VM (2004) De-ubiquitination and ubiquitin ligase domains of A20 downregulate NF-кB signalling. Nature 430: 694-699.

48. Rantala JK, Pouwels J, Pellinen T, Veltel S, Laasola P, Mattila E, Potter CS, Duffy T, Sundberg JP, Kallioniemi O, Askari JA, Humphries MJ, Parsons M, Salmi M, Ivaska J (2011) SHARPIN is an endogenous inhibitor of $\beta 1$-integrin activation. Nat Cell Biol 13: 1315-1324.

49. Elgueta R, Benson MJ, de Vries VC, Wasiuk A, Guo Y, Noelle RJ (2009) Molecular mechanism and function of CD40/CD40L engagement in the immune system. Immunol Rev 229: 152-172.

50. Hostager BS, Fox DK, Whitten D, Wilkerson CG, Eipper BA, Francone VP, Rothman PB, Colgan JD (2010) HOIL-1L interacting protein (HOIP) as an NF- $\kappa \mathrm{B}$ regulating component of the CD40 signaling complex. PLoS One 5: e11380.

51. Hostager BS, Kashiwada M, Colgan JD, Rothman PB (2011) HOIL-1L interacting protein (HOIP) is essential for CD40 signaling. PLoS One 6: e23061.

52. Takeuchi O, Akira S (2010) Pattern recognition receptors and inflammation. Cell 140: 805-820.

53. Zak DE, Schmitz F, Gold ES, Diercks AH, Peschon JJ, Valvo JS, Niemisto A, Podolsky I, Fallen SG, Suen R, Stolyar T, Johnson CD, Kennedy KA, Hamilton MK, Siggs OM, Beutler B, Aderem A (2011) Systems analysis identifies an essential role for SHANK-associated $\mathrm{RH}$ domain-interacting protein (SHARPIN) in macrophage Toll-like receptor 2 (TLR2) responses. Proc Natl Acad Sci U S A 108: 11536-11541.

54. Zhang M, Tian Y, Wang RP, Gao D, Zhang Y, Diao FC, Chen DY, Zhai ZH, Shu HB (2008) Negative feedback regulation of cellular antiviral signaling by RBCK1mediated degradation of IRF3. Cell Res 18: 1096-1104.

55. Inn KS, Gack MU, Tokunaga F, Shi M, Wong LY, K. 
Iwai K, Jung JU (2011) Linear ubiquitin assembly complex negatively regulates RIG-I- and TRIM25-mediated type I interferon induction. Mol Cell 41: 354-365.

56. Kato H, Takeuchi O, Sato S, Yoneyama M, Yamamoto M, Matsui K, Uematsu S, Jung A, Kawai T, Ishii KJ, Yamaguchi O, Otsu K, Tsujimura T, Koh CS, Reis e Sousa C, Matsuura Y, Fujita T, Akira S (2006) Differential roles of MDA5 and RIG-I helicases in the recognition of RNA viruses. Nature 441: 101-105.

57. Gack MU, Shin YC, Joo CH, Urano T, Liang C, Sun L, Takeuchi O, Akira S, Chen Z, Inoue S, Jung JU (2007) TRIM25 RING-finger E3 ubiquitin ligase is essential for RIG-I-mediated antiviral activity. Nature 446: 916920.

58. Seth RB, Sun L, Chen ZJ (2006) Antiviral innate immunity pathways. Cell Res 16: 141-147.

59. Jung J, Kim JM, Park B, Cheon Y, Lee B, Choo SH, Koh SS, Lee S (2010) Newly identified tumor-associated role of human Sharpin. Mol Cell Biochem 340: 161-167.

60. Tomonaga M, Hashimoto N, Tokunaga F, Onishi M, Myoui A, Yoshikawa H, Iwai K (2012) Activation of nuclear factor-kappa B by linear ubiquitin chain assembly complex contributes to lung metastasis of osteosarcoma cells. Int J Oncol 40: 409-417.

61. Miyamoto S (2011) Nuclear initiated NF-кB signaling: NEMO and ATM take center stage. Cell Res 21: 116130.

62. Huang TT, Wuerzberger-Davis SM, Wu ZH, Miyamoto S (2003) Sequential modification of NEMO/IKK $\gamma$ by SUMO-1 and ubiquitin mediates NF- $\kappa$ B activation by genotoxic stress. Cell 115: 565-576.

63. Jin HS, Lee DH, Kim DH, Chung JH, Lee SJ, Lee TH (2009) cIAP1, cIAP2, and XIAP act cooperatively via nonredundant pathways to regulate genotoxic stressinduced nuclear factor- $\kappa \mathrm{B}$ activation. Cancer Res 69: 1782-1791.

64. Hinz M, Stilmann M, Arslan SC, Khanna KK, Dittmar G, Scheidereit C (2010) A cytoplasmic ATM-TRAF6cIAP1 module links nuclear DNA damage signaling to ubiquitin-mediated NF- $\kappa \mathrm{B}$ activation. Mol Cell 40: 63-74.

65. Niu J, Shi Y, Iwai K, Wu ZH (2011) LUBAC regulates $\mathrm{NF}-\kappa \mathrm{B}$ activation upon genotoxic stress by promoting linear ubiquitination of NEMO. EMBO J 30: 37413753.

66. Boyce BF, Yao Z, Xing L (2010) Functions of nuclear factor $\kappa \mathrm{B}$ in bone. Ann N Y Acad Sci 1192: 367-375.

67. Xia T, Liang Y, Ma J, Li M, Gong M, Yu X (2011) Lossof-function of SHARPIN causes an osteopenic phenotype in mice. Endocrine 39: 104-112.

68. Landgraf K, Bollig F, Trowe MO, Besenbeck B, Ebert C, Kruspe D, Kispert A, Hanel F, Englert C (2010) Sipl1 and Rbck1 are novel Eya1-binding proteins with a role in craniofacial development. Mol Cell Biol 30: 57645775.

69. Wada T, Nakashima T, Hiroshi N, Penninger JM (2006) RANKL-RANK signaling in osteoclastogenesis and bone disease. Trends Mol Med 12: 17-25.

70. Baker RG, Hayden MS, Ghosh S (2011) NF-кB, inflammation, and metabolic disease. Cell Metab 13: 11-22. 